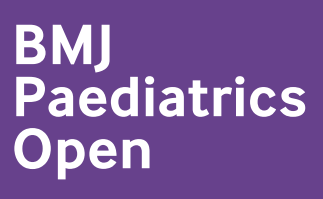

BMJ Open

\title{
Bowel preparation for elective procedures in children: a systematic review and meta-analysis
}

\author{
Morris Gordon, ${ }^{1,2}$ Fiona Karlsen, ${ }^{2}$ Sahira Isaji, ${ }^{2}$ Guan-Ong Teck ${ }^{2}$
}

To cite: Gordon M, Karlsen F, Isaji S, et al. Bowel preparation for elective procedures in children: a systematic review and meta-analysis. BMJ Paediatrics Open 2017;1:e000118. doi:10.1136/ bmjpo-2017-000118

- Additional material is published online only. To view please visit the journal online (http://dx.doi.org/10.1136/ bmjpo-2017-000118).

Received 29 May 2017 Revised 10 August 2017 Accepted 11 August 2017

\section{CrossMark}

${ }^{1}$ School of Medicine and Dentistry, University of Central Lancashire, Preston, UK ${ }^{2}$ Department of Paediatrics, Blackpool Victoria Hospital, Blackpool, UK

Correspondence to Dr Morris Gordon; Mgordon@ uclan.ac.uk

\section{ABSTRACT}

Objective Reviews have investigated preparation for colonoscopy, but not for surgery, They are also often limited to patients up to 16 years, despite many paediatric gastroenterologists caring for older patients. We carried out a systematic review investigating the optimum bowel preparation agents for all indications in children and young people.

Design A Cochrane format systematic review of randomised controlled trials (RCTs). Data extraction and assessment of methodological quality were performed independently by two reviewers. Methodological quality was assessed using the Cochrane risk of bias tool. Patients Young people requiring bowel preparation for any elective procedure, as defined by the primary studies. Interventions RCTs comparing bowel preparation with placebo or other interventions.

Main outcome measures Adequacy of bowel preparation, tolerability and adverse events.

Results The search yielded 2124 results and 15 randomised controlled studies ( $n=1435)$ but heterogeneity limited synthesis. Meta-analysis of two studies comparing polyethylene glycol (PEG) with sodium phosphate showed no difference in the quality of bowel preparation (risk ratio (RR) $1.27(95 \% \mathrm{Cl} 0.66$ to 2.44$))$. Two studies comparing sodium picosulfate/magnesium citrate with PEG found no difference in bowel preparation but significantly higher number of patients needing nasogastric tube insertion in the polyethylene glycol-electrolyte lavage solution (RR $0.04(95 \% \mathrm{Cl} 0.01$ to 0.18 ), 45 of 117 in PEG group vs 2 of 121 in sodium picosulfate group). Meta-analysis of three studies ( $n=241)$ found no difference between PEG and sennasoids (RR 0.73(95\% Cl 0.31 to 1.71)).

Conclusions The evidence base is clinically heterogeneous and methodologically at risk of bias. There is evidence that all regimens are equally effective. However, sodium picosulfate was better tolerated than PEG. Future research is needed with all agents and should seek to consider safety and tolerability as well as efficacy.

\section{BACKGROUND}

Bowel imaging is a crucial modality in the diagnosis and monitoring of inflammatory bowel disease, as well as surgery frequently being required in such patients. Multiple studies have suggested that bowel preparations must be individually tailored according

\section{What is already known on this topic?}

Bowel preparation is vital to imaging and surgery in young people, but it is key to consider tolerability as well as efficacy in these patients.

- Previous systematic reviews have only considered preparation for imaging and limit the age range, despite paediatricians often caring for children until older.

\section{What this study hopes to add?}

Despite many trials, there is much clinical heterogeneity and risk of bias concerns with the evidence base, as well as poor safety and tolerability reporting.

- There is evidence that polyethylene glycol (PEG) regimens are effective. However, when compared, sodium picosulfate was better tolerated than PEG.

- Future research needs to address these key methodology issues and also consider safety and tolerability as well as efficacy.

to patient age, size and clinical status. ${ }^{1}$ However, currently there is no internationally recognised gold standard regimen for paediatric bowel preparations. ${ }^{2}$ Several regimens have been tried with the aim of identifying the safest, efficacious and tolerable combination, with varying success. ${ }^{3}$

Bowel preparation regimens can be based on lavage (bowel clean out) or cathartics (agents that accelerates defecation). Examples include large volume of polyethylene glycol-electrolyte lavage solution (PEG-ELS) lavage solution, sodium phosphate (an oral, low-volume, hyperosmotic agent), sodium picosulfate, bisacodyl and dietary measures, such as diet packs or clear liquid diets (often in combination with other agents).

Adequate bowel preparation prior to such procedures is crucial to ensure complete visualisation of the colonic mucosa (thus successful diagnostic and therapeutic colonoscopy, endoscopy and capsule endoscopy) 
and to minimise the risk of possible contamination during surgery. Administration of the agents is much more problematic in children compared with adults who manage to take the agents readily. In uncooperative children, use of a nasogastric tube to administer the agents has been reported to be an effective method to guarantee bowel wash out. ${ }^{4}$ Reduced tolerance can result in poor outcomes due to inadequate preparation, increased rate of complications, extended procedural time and missed lesions. ${ }^{3}$ Additionally, side effects have previously been noted, such as hyperphosphataemia, in children who receive sodium phosphate. ${ }^{4}$

While previous reviews have considered preparation for colonoscopy, ${ }^{3}$ they do not always consider surgical interventions and often limit their populations at the age of 16 years, despite it being normal practice for paediatric gastroenterologists to look after such patients for a number of years before transition to adult services. ${ }^{5}$

We carried out an up-to-date systematic review using the Cochrane collaboration format to summarise the available evidence investigating the optimum bowel preparation agents for all indications in children and young people.

\section{METHODS}

The objectives of this review were to evaluate the efficacy and safety of different bowel preparation for young people for any indication. A full protocol for the study was completed by the authors prior to commencement of the study and is available on request. This set out the procedure for the search, study screening, data extraction, risk of bias evaluation and analysis.

\section{Criteria for considering studies for this review}

Randomised controlled trials (RCTs) were included in this systematic review. Participants were aged 0-21 years. This age range was selected after a scoping search and discussion with local stakeholders (tertiary centres) confirmed that it can be normal practice in paediatric gastroenterology for these patient groups to have variable transition to adult services, but 21 years was agreed as an absolute cut-off and reflected several studies that would otherwise be excluded. Studies with adults included that did not allow analysis of this. Paediatric age range was excluded. Studies compared bowel preparation with another bowel preparation or placebo, with all forms and dosing regimens considered. The purpose of bowel clearance was for colonoscopy or elective surgery. Studies were excluded if the purpose was to treat faecal impaction or encopresis. The primary outcome measure for the studies was the number of adequate bowel preparations, as defined by the included studies. Secondary outcomes included: tolerability (the proportion of children who could take the given therapy without the need for support through a nasogastric tube or incomplete dosing), duration of procedure, missed lesions due to inadequate bowel preparationand occurrence of any adverse events.

\section{Search methods for identification of studies}

Electronic searches (search strategy not limited by language) were completed using MEDLINE, The Cochrane Central Register of Controlled Trials, Embase and CINAHL (inception-15 July 2016) (online supplementary appendix 1). References of included trials were also searched. Manufacturers were contacted to identify further negative and unpublished research. Abstracts were considered for inclusion if full details to judge inclusion were offered or available from the authors after contact by the study team.

Data extraction and assessment of methodological quality of included studies were independently performed by two authors, and disagreements were solved with involvement of the third author.

\section{Data collection and analysis}

All identified abstracts and results from searches were reviewed by the authors. If the reference appeared relevant, a full copy of the study was obtained. After reading the full texts, each author independently assessed the eligibility of all trials identified based on the inclusion criteria above. Disagreement among authors was discussed, and agreement was reached by consensus. If the data to judge inclusion were unclear, attempts were made to contact the authors.

A data extraction form was developed and piloted to extract information on relevant features and results of all primary and secondary outcomes of included studies. The two reviewers separately extracted and recorded data on the predefined checklist, with disagreement discussed and consensus reached.

The risk of bias of selected trials was assessed independently by the authors using the Cochrane risk of bias tool with disagreement once again resolved by reaching consensus. Study authors were contacted for further information when insufficient information was offered to judge risk of bias or data were missing for primary outcomes. Analysis was completed using Revman (Review Manager 5.2, V.5.2.9, The Nordic Cochrane Centre, The Cochrane Collaboration, Copenhagen, Denmark, 2012).

The primary outcome-efficacy of bowel preparation agents-was assessed using the risk ratio (RR) with 95\% CI. The secondary outcomes were assessed by calculating the RR and $95 \% \mathrm{CI}$ or the mean difference (MD) with $95 \% \mathrm{CI}$, as indicated. The authors of included studies were again contacted to supply any missing data. Heterogeneity among trial results was assessed by inspection of graphical presentations and by calculating the $\chi 2$ test of heterogeneity (a p value of 0.10 was regarded as statistically significant). We also used the $\mathrm{I}^{2}$ statistic to quantity the effect of heterogeneity. ${ }^{6}$ A result of less than $25 \%$ was defined as low, up to $75 \%$ as moderate and above $75 \%$ as high heterogeneity. A random-effects model was used, with a sensitivity analysis with the fixed-effects model, 


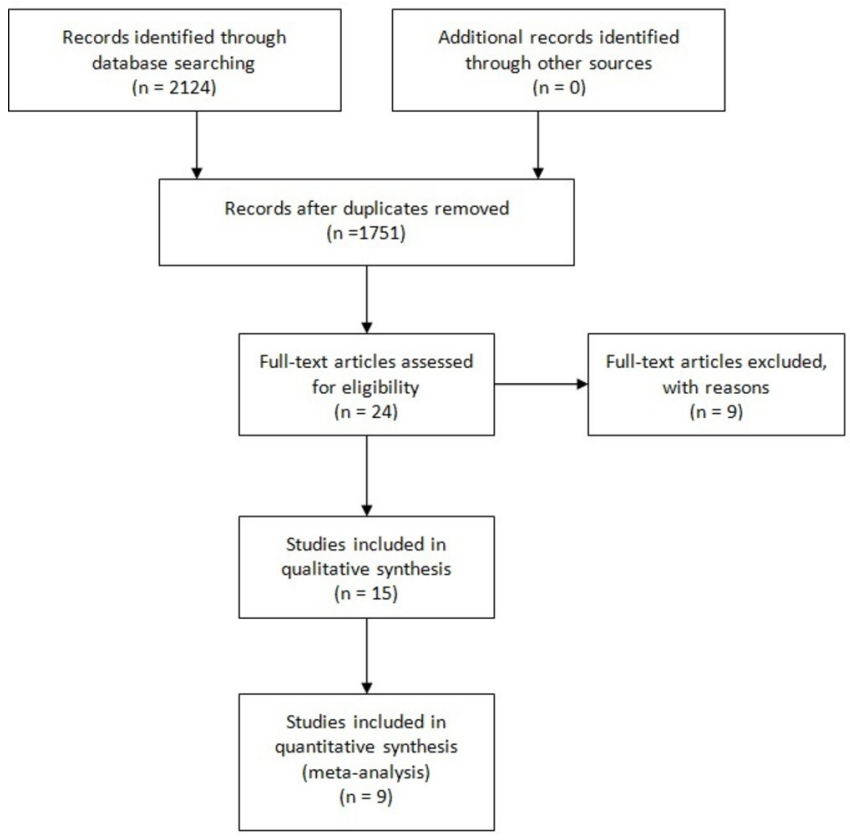

Figure 1 Patient flow diagram.

to identify differences in results that would suggest heterogeneity.

\section{RESULTS}

The electronic database search identified 2124 studies that were screened for inclusion. Of these, 15 studies $(n=1435)$ were judged to be potentially relevant and subjected to full-text review (figure 1). Only 12 papers needed consideration of a third author (less than $1 \%$ ) to reach consensus, with 1 included and 11 excluded. Experts were contacted, but no extra reports were received and no further studies were identified from drug companies.

\section{Description of studies Excluded studies}

Nine reports were excluded for failing to meet the inclusion criteria. Five were not solely with patients under 21 years of age, three were not RCTs and one was an abstract with insufficient data to judge inclusion.

\section{Included studies}

The 15 RCTs included described various regimens and comparative agents, with nine included in quantitative analysis (table 1) and the remaining six in qualitative analysis (table 2). Four studies compared various different regimens and combinations of polyethylene glycol (PEG), two compared PEG with oral sodium phosphate, two compared PEG with normal saline, three compared multiple combinations of PEG, sennasoids and sodium picosluphate, two diet kits with sodium phosphate, one study compared sodium picosulfate with phosphate enemas and one sodium picospulfate with PEG.
Risk of bias of included studies

Seven studies were rated as low risk for random sequence generation (selection bias) because these studies employed computer-generated randomisation. The remaining studies described themselves as randomised but, with no further details given or available from authors, were rated as unclear risk of bias.

Five studies were rated as low risk of bias for allocation concealment (selection bias). Nine remaining studies were rated as unclear risk of bias for allocation concealment as the methods were not clearly described in the manuscripts. One described the allocated researcher as performing colonoscopies and was rated as high risk.

Ten studies were blinded and were judged to be at low risk of bias for blinding of personnel (performance bias) for such an intervention. Four studies described themselves as blinded but gave no further details so was rated as unclear risk of bias. One study was open label and judged to be at high risk of bias for blinding.

Eight studies reported full and appropriate data and satisfactorily documented withdrawals and dropouts and were therefore judged to be at low risk of bias for incomplete outcome data (attrition bias), and nine were judged as low risk for selective reporting (reporting bias). Two studies did not record full data for all patients and were judged high risk of bias for attrition bias. Four studies did not offer outcome data regarding side effects and tolerability so were judged at high risk for reporting bias.

All studies were judged to be at low risk for other sources of bias. However, the small sample size of many of these studies is concerning, suggesting they were pilot or similarly underpowered studies, raising a further concern regarding bias. Details are summarised in table 1 .

The 15 studies present significant clinical and methodological heterogeneity (table 1 ), and this severely limits the scope for synthesis.

\section{PEG VERSUS SENNASOIDS}

Meta-analysis of three studies $(\mathrm{n}=241)^{7-9}$ found no difference between PEG and sennasoids in adequate bowel preparation (RR 0.73 (95\% CI 0.31 to 1.76), figure 2). High statistical heterogeneity was noted. Data regarding tolerability and safety were not presented to allow synthesis.

\section{Sodium picosulfate and magnesium citrate versus PEG-ELS}

Within these two studies versus PEG-ELS, ${ }^{10}{ }^{11}$ equivocal adequacy of bowel preparation was seen (RR 0.99 (95\% CI 0.89 to 1.11), figure 3). PEG's acceptability was reportedly poorer than sodium picosulfate in both studies. Meta-analysis of two PEG-ELS studies using the random effects model found a significantly higher number of patients needing nasogastric tube insertion in the PEG-ELS group (45 of 117) than the sodium picosulfate group (2 of 121) (RR 0.04 (95\% CI 0.01 to 0.18 ), figure 4). 


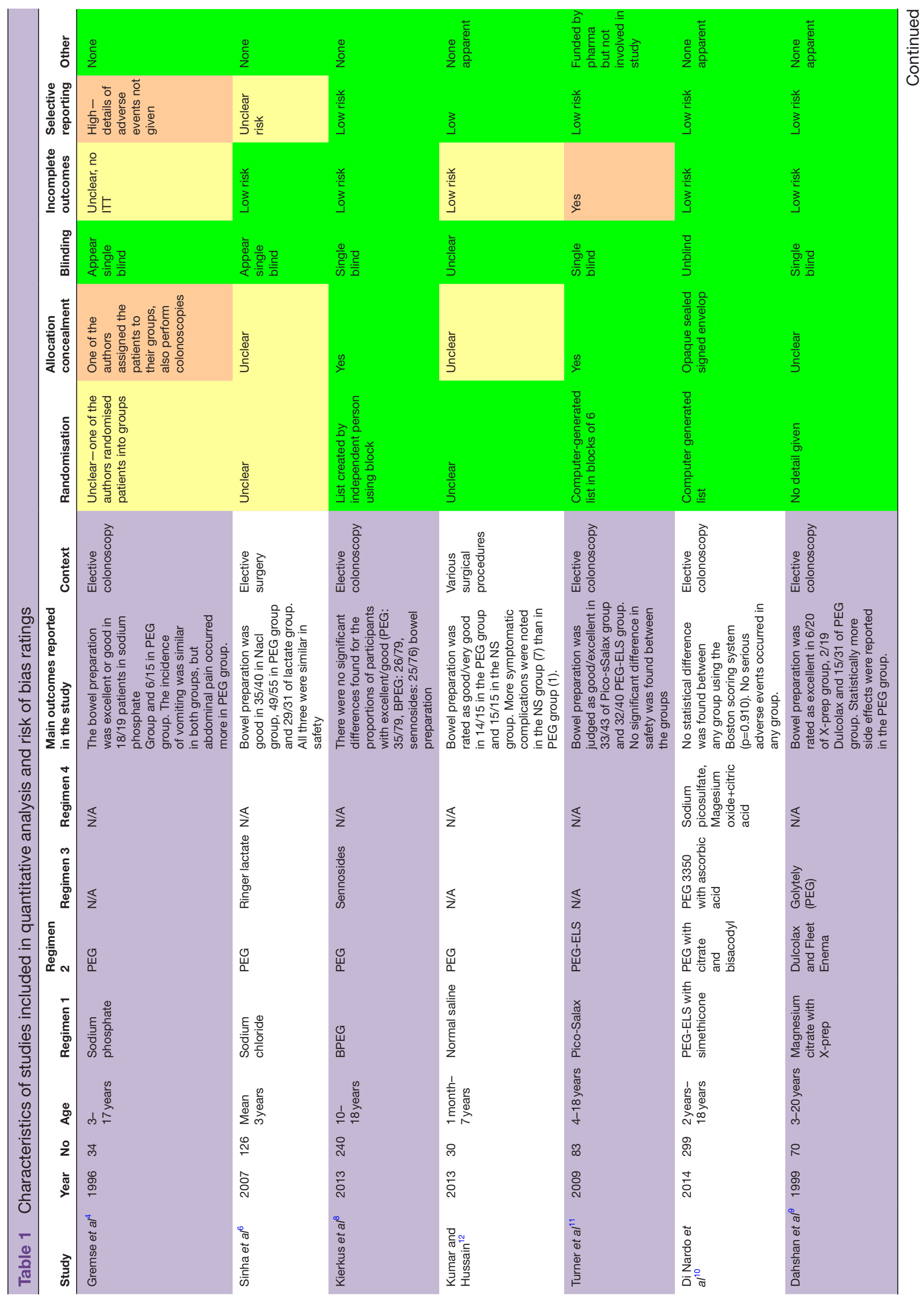




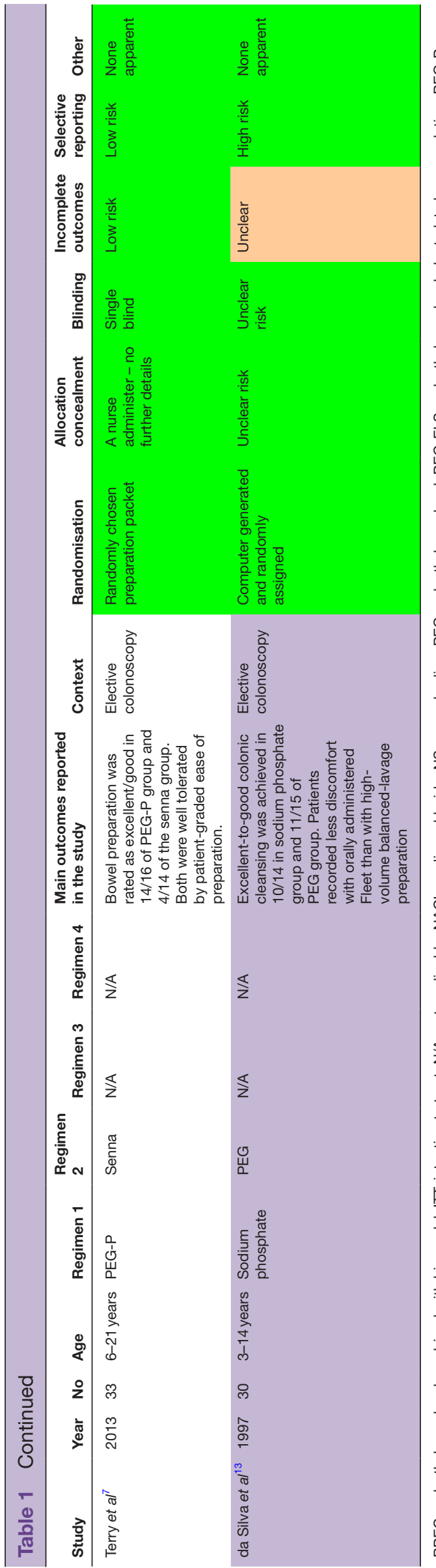

One patient in the PEG-ELS group and one in the sodium picosulfate group in the two studies were assessed as dehydrated and required intravenous fluids. For the PEG-ELS patient, a 10-year-old girl is reported who required intravenous fluid for 6 hours because of lethargy and dehydration (dryness of the oral mucosa and orthostatic hypotension) with serum electrolyte and glucose serum levels within the normal range. For the child in the sodium picosulfate group, a 12-year-old girl is reported that required intravenous fluids for 2 hours due to mild lethargy postprocedure and was discharged well thereafter. Her vital signs were always within normal limits, but her serum osmolality was $316 \mathrm{mOsm} / \mathrm{L}$; she had drunk only two glasses of apple juice during the entire duration of the bowel cleanout. No other serious adverse events were noted.

\section{PEG VERSUS NORMAL SALINE}

Meta-analysis of two studies $(\mathrm{n}=125)$ comparing PEG with normal saline ${ }^{612}$ found no difference in rate of adequate bowel preparation (RR 0.95 (95\% CI 0.87 to 1.04 ), figure 5). Adverse events were not reported homogeneously to allow analysis, but occurred in both groups, including abdominal pain and vomiting.

\section{PEG VERSUS SODIUM PHOSPHATE}

There were two studies concerning 63 participants. ${ }^{4}{ }^{13}$ Meta-analysis of two studies using the random-effect model found no difference in the adequacy of bowel preparation (RR 1.27 (95\% CI 0.66 to 2.45), figure 6). Again, high statistical heterogeneity was noted. One of the studies needed to insert a nasogastric tube in all patients receiving PEG, while in the remaining study, $53 \%$ of participants in the PEG group were unable to finish taking the solution, while all the patients in the sodium phosphate group could complete the medication. As these were reported differently, no meta-analysis was performed. No serious adverse events were reported.

\section{OTHER STUDIES}

Within the remaining studies, ${ }^{14-18}$ no meta-analysis was possible. However, no individual study found any different in adequacy of bowel preparation or adverse events. Tolerability was not well reported across studies. While secondary outcome analysis for further items were planned, data were not presented to allow this to take place.

\section{DISCUSSION}

Despite the common requirement for bowel preparation in young people, the results of this review have highlighted a very poor evidence base. A mixture of clinical heterogeneity related to multiple agent regimens and methodological heterogeneity limiting the ability 


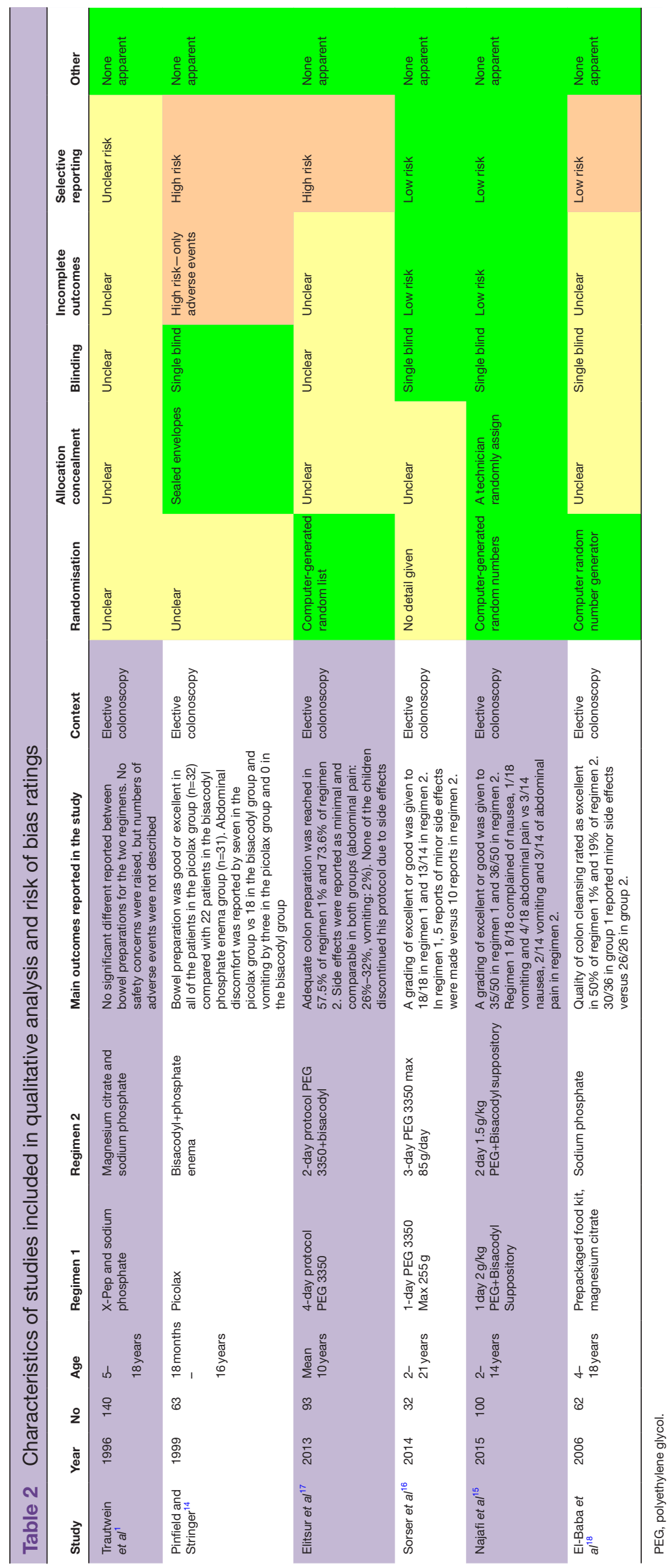




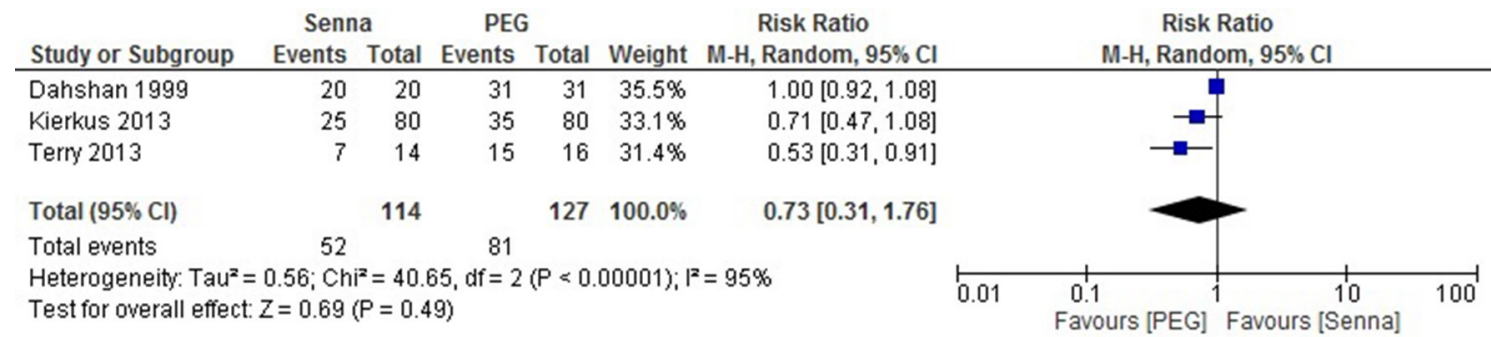

Figure 2 Forest plot for PEG versus senna: adequacy of bowel preparation. PEG, polyethylene glycol.

for meta-analysis has significantly limited synthesis. In multiple small analyses, PEG-ELS, senna, normal saline, sodium phosphate and sodium picosulfate/magnesium citrate found no difference in adequacy of bowel preparation. This was similar across the remaining individual studies. As such, despite the significant weaknesses of the evidence base, it is worth noting that no difference in adequacy of bowel preparation has been reported in any included study. This was also the case with adverse events, although it must be noted these were reported in an extremely heterogeneous fashion, with individual minor, major and patient overall recording of events across studies.

Of note, there was a significant difference in favour of sodium picosulfate and magnesium citrate regarding tolerability, specifically the need for a nasogastric tube to complete the bowel preparation. This is a particularly interesting finding, as the primary studies highlighted that while tolerability of PEG was extremely poor, smaller volumes than planned appeared to have little impact on efficacy. This raises the question of the need for the nasogastric tube at all and so this may need further investigation in the future. Sodium picosulfate was also compared with bisacodyl and a phosphate enema in a single study with equivocal preparation, tolerability and safety reported.
PEG appeared to be the least tolerable agent across all studies, with a number of the patients requiring a nasogastric tube insertion, but this is from qualitative synthesis of individual studies, with this outcome reported in heterogeneous fashion so meta-analysis was not possible. Additionally, as the age ranges of included participants varied greatly, it is hard to make firm conclusions on this finding as the need for nasogastric tubes is likely to be very age dependent. Oral sodium phosphate was well tolerated in individual studies. Despite the satisfactory tolerability and safety profile of sodium phosphate, it should be noted that care must be taken when using this agent as it can cause significant electrolyte imbalances. As such, it should not be used in patients with deranged baseline electrolytes, suboptimal renal and hepatic function, as it poses a risk of acute kidney injury and phosphate nephropathy. ${ }^{19}$

With all the agents studied, the occurrence of minor adverse events such as abdominal pain, bloating, faecal incontinence, nausea, vomiting, headaches and anal irritation was comparable. No serious adverse events were reported in any of the studies. It should also be noted that in the context of elective surgery, there is growing recognition of the role for proceeding without bowel preparation. ${ }^{20}$ 


\begin{tabular}{|c|c|c|c|c|c|c|c|c|}
\hline \multirow[b]{2}{*}{ Study or Subgroup } & \multicolumn{2}{|c|}{ Normal saline } & \multicolumn{2}{|c|}{ PEG } & \multirow[b]{2}{*}{ Weight } & \multirow{2}{*}{$\begin{array}{c}\text { Risk Ratio } \\
\text { M-H, Random, } 95 \% \mathrm{Cl}\end{array}$} & \multirow{2}{*}{$\begin{array}{l}\text { Risk Ratio } \\
\text { M-H, Random, } 95 \% \mathrm{Cl}\end{array}$} & \\
\hline & Events & Total & Events & Total & & & & \\
\hline Kumar 2013 & 14 & 15 & 15 & 15 & $24.2 \%$ & $0.94[0.78,1.12]$ & & \\
\hline Sinha 2007 & 37 & 40 & 53 & 55 & $75.8 \%$ & $0.96[0.87,1.06]$ & & \\
\hline Total $(95 \% \mathrm{Cl})$ & & 55 & & 70 & $100.0 \%$ & $0.95[0.87,1.04]$ & & \\
\hline & 51 & & 68 & & & & & \\
\hline \multicolumn{7}{|c|}{$\begin{array}{l}\text { Heterogeneity: } \text { Tau }^{2}=0.00 ; \mathrm{Chi}^{2}=0.06, \mathrm{df}=1(\mathrm{P}=0.81) ; \mathrm{I}^{2}=0 \% \\
\text { Test for overall effect: } Z=1.04(P=0.30)\end{array}$} & $\begin{array}{lcr}1 & 0.85 & 1 \\
& \text { Favours [PEG] } & 1.2 \\
& \text { Favours [ }\end{array}$ & $\begin{array}{c}1.5 \\
\text { al saline] }\end{array}$ \\
\hline
\end{tabular}

Figure 6 Forest plot for PEG versus sodium phosphate: adequacy of bowel preparation. PEG, polyethylene glycol.

The evidence base for this review covers a large number of trials with a reasonable number of patients but is severely limited due to clinical and methodological heterogeneity, as well as concerns with risk of bias. As such, the findings of this review should be interpreted with extreme caution as it is difficult to draw firm conclusions for any of the investigated agents. It must also be noted that, for the primary outcome, successful bowel preparation was 'as defined" by primary studies, with several different scoring systems and criteria used. This also limits the appropriateness of meta-analysis in this context, although those wishing to complete future studies should note the Ottawa scoring system ${ }^{21}$ was the only such scoring method reported in multiple studies. This is also true of adverse events, which were reported in a sporadic and inconsistent manner that prevented comment on even simple complaints, such as nausea or vomiting.

Considering the small sample sizes, the high degree of heterogeneity and a wide variation in the regimen of each cleansing agent, the findings of this review cannot be reliably used to inform clinical practise but most usefully should inform future research. In particular, as the question of adequacy of bowel preparation has been established as essentially equivocal among all study agents, a shift of focus for future studies is needed. Given the unique needs of a paediatric population, considering the issue of tolerability as a primary outcome is vital, and looking at the lower volume options presented as enteral agents could offer potential practical advantages and need a high-quality study to investigate them.

\section{CONCLUSIONS}

The publishing evidence base investigating this issue is large but is clinically heterogeneous and at risk of bias. All regimens appear equivocal for adequacy of bowel preparation. However, when compared with sodium picosulfate, sodium picosulfate is better tolerated. Future research should seek to consider safety and tolerability, as well as efficacy, given the key importance of these issues in a childhood population.

Contributors All authors contributed to and approved the final manuscript. MG conceived the project, supported analysis and contributed in writing the paper. SI and FK led the search, extraction and analysis. G-OT led the analysis and write-up.

Competing interests None declared.

Provenance and peer review Not commissioned; externally peer reviewed.

Data sharing statement All data available on request.

Open Access This is an Open Access article distributed in accordance with the Creative Commons Attribution Non Commercial (CC BY-NC 4.0) license, which permits others to distribute, remix, adapt, build upon this work non-commercially, and license their derivative works on different terms, provided the original work is properly cited and the use is non-commercial. See: http://creativecommons.org/ licenses/by-nc/4.0/

(c) Article author(s) (or their employer(s) unless otherwise stated in the text of the article) 2017. All rights reserved. No commercial use is permitted unless otherwise expressly granted.

\section{REFERENCES}

1. Trautwein AL, Vinitski LA, Peck SN. Bowel preparation before colonoscopy in the pediatric patient: a randomized study. Gastroenterol Nurs 1996;19:137-9.

2. Engum SA, Carter ME, Murphy D, et al. Home bowel preparation for elective colonic procedures in children: cost savings with quality assurance and improvement. J Pediatr Surg 2000;35:232-4.

3. Pall H, Zacur GM, Kramer RE, et al. Bowel preparation for pediatric colonoscopy: report of the NASPGHAN endoscopy and procedures committee. J Pediatr Gastroenterol Nutr 2014;59:409-16.

4. Gremse DA, Sacks Al, Raines S. Comparison of oral sodium phosphate to polyethylene glycol-based solution for bowel preparation for colonoscopy in children. $J$ Pediatr Gastroenterol Nutr 1996;23:586-90.

5. Hunter A, Mamula P. Bowel preparation for pediatric colonoscopy procedures. J Pediatr Gastroenterol Nutr 2010;51:1-61.

6. Sinha SK, Kanojia RP, Rawat JD, et al. Comparison of three solutions for total gut irrigation in pediatric patients. Pediatr Surg Int 2007;23:581-4.

7. Terry NA, Chen-Lim ML, Ely E, et al. Polyethylene glycol powder solution versus senna for bowel preparation for colonoscopy in children. J Pediatr Gastroenterol Nutr 2013;56:215-9.

8. Kierkus J, Horvath A, Szychta M, et al. High- versus low-volume polyethylene glycol plus laxative versus sennosides for colonoscopy preparation in children. J Pediatr Gastroenterol Nutr 2013;57:230-5. 
9. Dahshan A, Lin $\mathrm{CH}$, Peters J, et al. A randomized, prospective study to evaluate the efficacy and acceptance of three bowel preparations for colonoscopy in children. Am J Gastroenterol 1999;94:3497-501.

10. Di Nardo G, Aloi M, Cucchiara S, et al. Bowel preparations for colonoscopy: an RCT. Pediatrics 2014;134:1542:249-56.

11. Turner D, Benchimol El, Dunn $\mathrm{H}$, et al. Pico-Salax versus polyethylene glycol for bowel cleanout before colonoscopy in children: a randomized controlled trial. Endoscopy 2009;41:1038-45.

12. Kumar A, Hussain A. Preoperative bowel preparation in children: polyethylene glycol versus normal saline. Afr J Paediatr Surg 2013;10:235-8.

13. da Silva MM, Briars GL, Patrick MK, et al. Colonoscopy preparation in children: safety, efficacy, and tolerance of high- versus low-volume cleansing methods. J Pediatr Gastroenterol Nutr 1997;24:33-7.

14. Pinfield A, Stringer MD. Randomised trial of two pharmacological methods of bowel preparation for day case colonoscopy. Arch Dis Child 1999;80:181-3.

15. Najafi M, Fallahi GH, Motamed F, et al. Comparison of one and two-day bowel preparation with polyethylene glycol in pediatric colonoscopy. Turk J Gastroenterol 2015;26:232-5.
16. Sorser SA, Konanki V, Hursh A, et al. 1-day bowel preparation with polyethylene glycol 3350 is as effective and safe as a 3-day preparation for colonoscopy in children. BMC Res Notes 2014;7:648.

17. Elitsur R, Butcher L, Vicki L, et al. Polyethylene glycol 3350 based colon cleaning protocol: $2 \mathrm{~d}$ vs $4 \mathrm{~d}$ head to head comparison. World $J$ Gastrointest Endosc 2013;5:165-8.

18. El-Baba MF, Padilla M, Houston C, et al. A prospective study comparing oral sodium phosphate solution to a bowel cleansing preparation with nutrition food package in children. $J$ Pediatr Gastroenterol Nutr 2006;42:174-7.

19. US Food and Drug administration (FDA). FDA warns of possible harm from exceeding recommended dose of over-the-counter sodium phosphate products to treat constipation. $2014 \mathrm{http} / / \mathrm{www}$. fda.gov/downloads/Drugs/DrugSafety/UCM381084.pdf.

20. Shah M, Ellis CT, Phillips MR, et al. Preoperative bowel preparation before elective bowel resection or ostomy closure in the pediatric patient population has no impact on outcomes: A Prospective Randomized Study. Am Surg 2016;82:801-6.

21. Rostom A, Jolicoeur E. Validation of a new scale for the assessment of bowel preparation quality. Gastrointest Endosc 2004;59:482-6. 